\title{
Structural modifications of quasi-one-dimensional submonolayers by impurity doping
}

\author{
G. Godzik, T. Block, and H. Pfnür* \\ Institut für Festkörperphysik, Universität Hannover, Appelstrasse 2, D-30167 Hannover, Germany \\ (Received 17 December 2003; revised manuscript received 24 March 2004; published 28 June 2004)
}

\begin{abstract}
In this study, carried out by analysis of intensities and spot profiles in low energy electron diffraction, we investigated the influence of electronegative oxygen atoms as dopants on the long range order, the thermal stability, and the type of phase transitions in the strongly anisotropic chain system $\mathrm{Sr}$ on $\mathrm{Mo}(112)$ in the low coverage regime at constant $\mathrm{Sr}$ concentrations up to 0.2 monolayers (ML), where the pure Sr layers show $p(8 \times 1)$ and $p(5 \times 1)$ structures, respectively. A single oxygen atom always influences many unit cells of $\mathrm{Sr}$, as expected from a model of coupling the $\mathrm{Sr}$ chains by Friedel oscillations. Whereas concentrations of only $0.02 \mathrm{ML}$ of oxygen transform the $p(8 \times 1)$ into a $p(9 \times 1)$ structure, small concentrations of oxygen atoms stabilize, but do not alter the $p(9 \times 1)$ structure over a wide range of oxygen concentrations. Oxygen induced disappearance of depinning transitions was found already for concentrations above 0.01 ML. For these concentrations only melting transitions are still observed. In a concentration range of oxygen and $\mathrm{Sr}$ atoms between 1:2 and 1:1 incommensurate structures are formed yielding finally islands of a $(3.3 \times 1)$ structure in all cases, interpreted as a sign of beginning formation of mixed oxides. Implications for adsorbate induced structuring of templates are discussed.
\end{abstract}

DOI: $10.1103 /$ PhysRevB.69.235414

PACS number(s): 64.70.Rh, 68.35.Rh

\section{INTRODUCTION}

Structuring by self-organized processes is particularly attractive in reduced dimensionality, e.g., on surfaces of single crystals. Here structuring is possible both on the true nanoscale, i.e., on the scale of a few atomic distances, but also on mesoscopic scales. ${ }^{1}$ Whereas the latter may involve destabilization of certain orientations of surfaces, e.g., by adsorbed layers at various concentrations, ${ }^{2,3}$ the former are governed by structures formed by the adsorbate itself. The chain structures investigated in the study presented here are examples of this type of structuring. Due to coupling between the adsorbed particles, mostly by indirect interactions through the substrate, various long range ordered structures can be adjusted and sometimes simply be tuned by concentration. ${ }^{4}$ Strongly anisotropic surfaces, or surfaces with an adsorbate induced reduction of symmetry, are particularly interesting in this context. ${ }^{5}$

As will be shown below, adsorbed atoms form chain structures on a variety of such surfaces, which seem to be coupled by adsorbate induced one-dimensional Friedel oscillations that screen the electronic distortion of the chemical bond between adsorbate and substrate. The characteristic wavelength of this screening is the Fermi wavelength. On a surface with large contributions by surface states, the effective electron density at the surface may be modified by adsorbates, thus changing the effective wavelength. This type of coupling offers large flexibility and opens a wide range of possibilities for manipulation. It is the purpose of this paper to show to which extent such a model is realistic.

We use for our investigations $\mathrm{Sr}$ atoms adsorbed on Mo(112), which form, as many electropositive elements on channeled metal surfaces, linear $p(n \times 1)$ chain structures at low coverages ${ }^{6}$ with $n$ up to 9 . Contrary to the structurally similar (110) surfaces of transition metals with a fcc crystal structure like $\mathrm{Au}$ or $\mathrm{Pt}$, the $\mathrm{Mo}(112)$ surface is not reconstructed. ${ }^{7}$ Apart from the formation of strongly coupled chains, which is a local electronic effect, the coupling mechanism between chains over distances up to 8 or even 9 lattice constants and the relatively high thermal stability ${ }^{8}$ becomes visible by the adsorbate induced vertical and lateral relaxation, which have been investigated in detail by low energy electron diffraction (LEED)-IV analysis for $\mathrm{Li}$ and $\mathrm{Sr}$ on Mo(112). ${ }^{9,10}$ Wavelike modulations of the substrate density in the topmost three substrate layers have been found that are induced by the adsorbate. These modulations may be explained by coupling of lattice relaxations and the electron density at the Fermi surface. Friedel oscillations, induced by the distortions of the electron density due to the adsorbate, can couple with the lattice and cause a lateral interaction potential also between the adsorbate chains. Surface states crossing the Fermi level have indeed been found in density functional calculations on the clean surface of $\operatorname{Mo}(112){ }^{11}$ Since the electron density of states close to the Fermi level from bulk states is low in the topmost substrate layers, as shown in Ref. 11, the influence of surface states actually dominates. Although our previous experiments and those described here can provide only indirect evidence, a plausible physical scenario is that the periodicity of the chain structure of the adsorbate as well as the adsorbate induced relaxations in the substrate are stabilized by adsorbate induced charge density waves in these systems. ${ }^{4,5,12-15}$ The Fermi wave vector can indeed be effectively tuned by the use of different adsorbates and by their concentration, as shown for $\mathrm{Li}$ and $\mathrm{Sr}$, which form ordered structures with different chain separations.

In this paper we extend a recent study that concentrated on the thermodynamic properties of the pure $\mathrm{Sr} / \mathrm{Mo}(112)$ system at coverages up to 0.2 monolayers (ML). ${ }^{16}$ In order to test the proposed coupling mechanism between chains-a mixture of dipole-dipole repulsion and an oscillatory part due to the charge density waves ${ }^{10}$ - but also to demonstrate its limitations, we added electronegative atomic oxygen to 
the ordered structures of electropositive $\mathrm{Sr}$ at low coverages. We show that, contrary to the generally accepted role of impurities to destroy long range order, oxygen is able to even stabilize and modify long range order at concentrations far below one atom per unit cell. In addition, it also modifies the observed phase transitions quantitatively so that the two predicted transitions from a commensurate-incommensurate transition to a striped soliton phase by formation of domain walls and a second transition of continuous melting of these solitons by unbinding of pairs of kinks ${ }^{17}$ merges into one transition.

\section{EXPERIMENT}

To perform the experiments, we used the UHV system and the experimental setup described earlier. ${ }^{8,16,18,19}$ The base pressure in the vacuum chamber between 0.7 and 1.5 $\times 10^{-11}$ mbar was kept all the time, i.e., both during deposition and subsequent measurements.

The same molybdenum sample as in our previous studies $^{8,19}$ was used as substrate, which is characterized by average (112) terrace sizes of $350 \AA \times 550 \AA$ along the [11 $\overline{1}]$ and $[1 \overline{1} 0]$ axes, respectively. The Mo(112) sample was routinely cleaned by many heating cycles in oxygen between 800 and $2000 \mathrm{~K}$. Oxygen was finally removed by several heating cycles to $2400 \mathrm{~K}$ in vacuum. Auger electron spectroscopy was used to check the purity of the substrate and adsorbate. The coverage $\theta$ is defined as the ratio of adsorbate to substrate surface atom concentrations. For both $\mathrm{Sr}$ and oxygen it was determined from the deposition time and from work function measurements calibrated by means of LEED in the coverage range $\theta=0-0.5 \mathrm{ML}$ for pure layers using the maximum intensities of the various LEED structures of both oxygen, ${ }^{20,21}$ here in particular the $(2 \times 1)$ structure at a coverage of 0.5, and the well-known Sr LEED structures. ${ }^{8}$ In this range of coverage the relation between the coverage and LEED pattern is unique. With the contact potential difference (CPD) method we tested that the work function changes were additive for the mixtures and did not depend on the sequence of adsorption. The same work function changes have been observed for fixed exposures also in the mixed layers so that we can safely assume that the sticking coefficients are unchanged with respect to the pure layers for this system. The flux of the Sr source, described below, could be held constant within a few percent over hours, so that coverages on the surface were determined by exposure times with a precision of less than $5 \%$ for all coverages.

An atomic-beam source of $\mathrm{Sr}$ was built by thermal activation in UHV of $\mathrm{SrO}_{2}$ mixed with $\mathrm{Al}$ powder. ${ }^{22}$ Deposition of the adsorbates $\mathrm{Sr}$ and oxygen was carried out at the lowest available substrate temperature $(80 \mathrm{~K})$. Measurements were only started after annealing these layers to $700 \mathrm{~K}$. Each annealing step lasted $30 \mathrm{~s}$ and was followed by a gradual temperature decrease at a rate of $-4 \mathrm{~K} / \mathrm{s}$ back to the base temperature.

The method of electron diffraction, used for all our measurements, directly yields the thermodynamic averages of interest. These would be almost impossible to derive with local
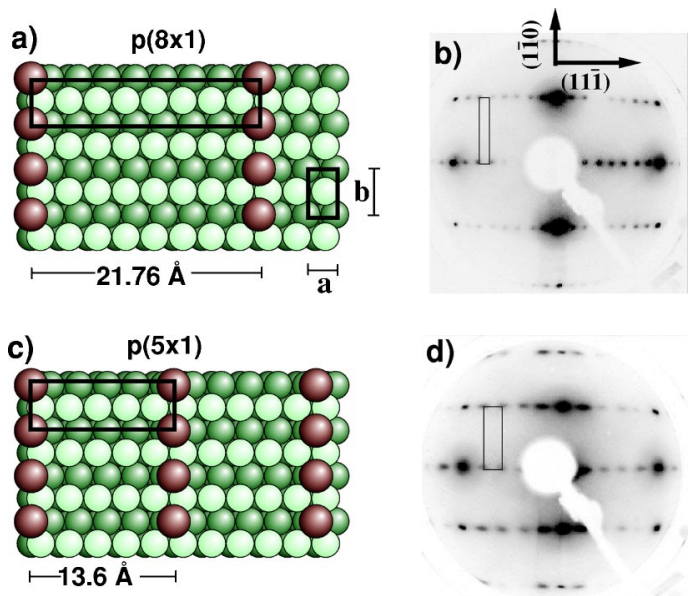

FIG. 1. (Color online) Real and reciprocal space images of the Sr induced low-coverage commensurate structures $p(8 \times 1)$ and $p(5 \times 1)$. Unit cells of the substrate and the superstructures are marked.

probes like tunneling microscopy for the large unit cells of the $p(8 \times 1), p(5 \times 1)$, and $p(9 \times 1)$ structures, which correspond to saturation coverages of 0.125 and $0.2 \mathrm{ML}$, respectively. The LEED patterns of these structures together with real space models are shown in Fig. 1. A SPECTALEED instrument equipped with a slow scan charge coupled device camera was used for studying the surface structure. The stored images of the LEED patterns were used to obtain the peak intensities and full widths at half maximum (FWHM) for the particular spots. Data were taken at intervals of $4 \mathrm{~s}$ during heating and cooling at a rate of $0.2 \mathrm{~K} / \mathrm{s}$ (between 400 and $600 \mathrm{~K}, 0.5 \mathrm{~K} / \mathrm{s}$ ).

\section{RESULTS AND DISCUSSION}

\section{A. Doping of oxygen atoms to the $p(8 \times 1)$ layer of $\mathrm{Sr}$}

We start with investigations of modifications of the $\mathrm{Sr}$ induced $p(8 \times 1)$ structure, which corresponds to an optimal coverage of $0.125 \mathrm{ML}$, by doping it with oxygen. In Fig. 2 we show a section of the LEED pattern of the $p(8 \times 1)$ structure at a constant $\mathrm{Sr}$ coverage of 0.11 as a function of oxygen

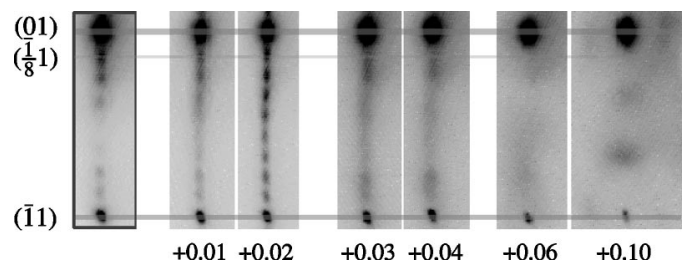

FIG. 2. Sections of the LEED pattern $\left(E_{\text {primary }}=80 \mathrm{eV}\right.$, surface temperature $70 \mathrm{~K}$ ) between $(01)$ and $(\overline{1} 1)$ spots at various concentrations of oxygen added to a constant $\mathrm{Sr}$ coverage of $0.11 \mathrm{ML}$. The leftmost pattern is that of a pure Sr layer at this coverage. For better orientation, thick gray lines mark the positions of the integer order beams, the thin line that of the $\left(\frac{\bar{T}}{8} 1\right)$ spot. Note the positional changes of the superstructure spots as a function of oxygen concentration. 


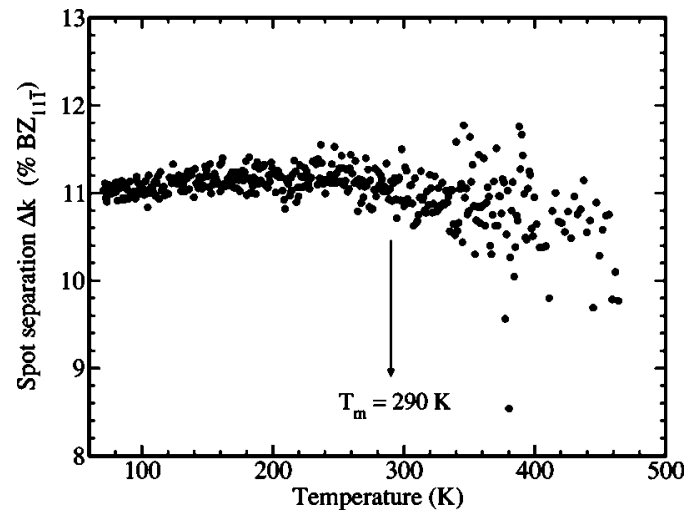

FIG. 3. Position of the $\left(\frac{\overline{2}}{9} 1\right)$ spot in $k$ space as a function of surface temperature after adsorption of $0.11 \mathrm{ML}$ of $\mathrm{Sr}$ and $0.02 \mathrm{ML}$ of oxygen.

concentration. This section between the (01) and the (11) beam contains all information about periodic structural changes, since these happen only unilaterally in the [1ㅣㅣ direction. For reasons of presentation, those sections that are horizontal in Fig. 1 are arranged vertically here.

Only the very small concentration of oxygen impurity of $1 \%$ of a monolayer $(1 \% \mathrm{ML})$ has the typical impurity effect: It disturbs the original order, as visible in the further reduction of intensity of the higher order fractional beams. The addition of $0.02 \mathrm{ML}$, however, has the opposite effect. It generates new long range order and changes the original $p(8 \times 1)$ structure to a $p(9 \times 1)$ structure. This concentration of oxygen doping yields the best long range order for this structure, as also visible by it highest thermal stability compared with other oxygen concentrations.

The phase transitions at a constant $\mathrm{Sr}$ coverage of 0.11 and at various concentrations of oxygen between 0 and 0.04 ML have been determined using both spot profiles and integral intensities [an example for the extraction of transition temperatures, etc., will be shown below for the $p(5$ $\times 1)$ structure]. For the pure Sr layer, two small anomalies were observed in the integral intensities as a function of temperature, as described in detail in Ref. 16 for the pure system. The anomaly close to $96 \mathrm{~K}$ was attributed to a depinning transition of the $\mathrm{Sr}$ chain lattice, since it was found to be correlated with the onset of a change of the average lattice constant, ${ }^{16}$ whereas the anomaly at $197 \mathrm{~K}$ marks melting of the chain lattice, indicated by a significant acceleration of the loss of long range correlations mainly in the [1 $1 \overline{1} 0]$ direction. These results for the $p(8 \times 1)$ structure of the pure $\mathrm{Sr}$ system are included in the phase diagram (see Fig. 5 below).

The addition of small amounts of oxygen to the $p(8 \times 1)$ ordered Sr layer eliminates the observation of a depinning transition already for the smallest oxygen concentration of $0.01 \mathrm{ML}$. This can be clearly seen from Fig. 3, where the position of the $\left(\frac{2}{9} 1\right)$ superstructure spot after additional adsorption of $0.02 \mathrm{ML}$ of oxygen is plotted as a function of temperature. As can be seen from this figure, the position of this spot is unchanged (within limits of uncertainty) up to $290 \mathrm{~K}$, where the only anomaly in the integrated intensity is

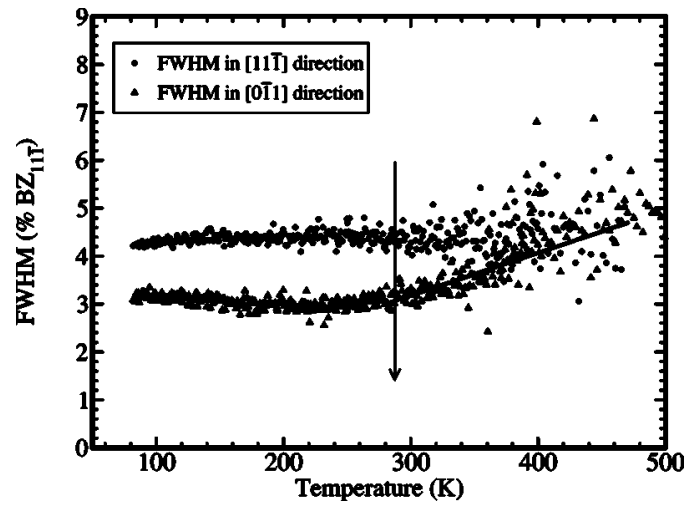

FIG. 4. Sr- $p(8 \times 1)+0.02$ ML oxygen: Full widths at half maximum (FWHM) of the $\left(\frac{\overline{2}}{9} 1\right)$ spot in $11 \overline{1}$ and in $0 \overline{1} 1$ directions for the same conditions as in Fig. 3.

seen (not shown). From the constant position up to this temperature we conclude that, in contrast to the pure Sr system, no depinning transition occurs. Instead the order of the $p(9$ $\times 1)$ structure persists up to this temperature, as seen from the temperature behavior of the half-widths, shown in Fig. 4. Different from the pure $\mathrm{Sr}$ system, which exhibits a gradual increase of the FWHMs above the depinning transition, these stay constant below $290 \mathrm{~K}$ in the oxygen doped system, fol-

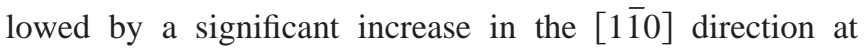
higher temperatures, again an indication that no phase transition occurs below this temperature. Similar to the pure $\mathrm{Sr}$ system at this coverage, the half-widths in the [111] direction change little at temperatures above melting, indicating that the chain separation of 9 lattice constants ( 8 in the pure $\mathrm{Sr}$ system) is strongly favored even at high temperatures.

The change of the average chain separation from 8 to 9 lattice constants induced by small concentrations of oxygen, together with a constant overall density of $\mathrm{Sr}$, implies that there is an excess coverage of $\mathrm{Sr}$ on the surface that has to be accommodated within the new ordered structure. This could be accomplished, e.g., either by introducing point defects into the layer or by doubling some of the chains. LEED, especially at these very low concentrations, has only a limited ability to discriminate between the various possibilities.

The results of temperature driven phase transitions, derived from integral LEED intensities of superstructure beam intensities, as described above, for a constant $\mathrm{Sr}$ concentration of $0.11 \mathrm{ML}$, but varying concentrations of oxygen atoms, are summarized in the phase diagram shown in Fig. 5. It emphazises two effects: First, any depinning transitions are removed already by traces of oxygen, and for all coverages of oxygen. Second, the new ordered $p(9 \times 1)$ structure that appears is induced by a rather narrow range of oxygen concentrations. These small concentrations of oxygen in fact exclude the possibility that there is a correlation between the oxygen atoms. If they would form islands with any kind of structure, this would exclude any influence on the whole average lattice constant of the adsorbed Sr layer. A correlation of oxygen with the $\mathrm{Sr}$ chains, however, is possible, but not detectable with LEED at these concentrations. In any case, we note that the adsorption of traces of oxygen influences the 


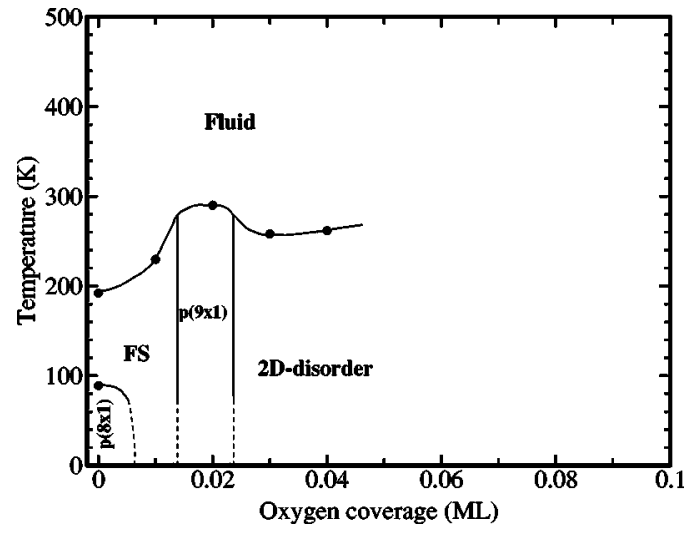

FIG. 5. Phase diagram induced by a varying oxygen concentration as indicated, determined for a constant $\mathrm{Sr}$ coverage of 0.11 ML. Lines mark experimentally observed ranges of stability with exact transition temperatures determined at some coverages (dots), whereas dashed lines complete the phase diagram in experimentally inaccessible temperature ranges. FS means floating solid.

average correlations of the whole layer so that the effect of oxygen is not just local.

The $p(9 \times 1)$ structure is in fact the only oxygen induced long range ordered structure in this adsorbate system. It is also remarkable that oxygen not only induces this new ordered structure, but also that the thermal stability of this structure is strongly enhanced compared with the $p(8 \times 1)$ structure of the clean $\mathrm{Sr}$ system. Oxygen concentrations above $0.03 \mathrm{ML}$, however, lead to a deterioration of order and, above a concentration of $0.06 \mathrm{ML}$, to the formation of very small ordered patches with a $(3.3 \times 1)$ structure, as seen from Fig. 2. This formation of short range correlations can be regarded as an indication for the beginning of oxide formation once a ratio between oxygen and $\mathrm{Sr}$ atoms of 1:2 is reached or exceeded. This oxidic species is strongly influenced by the presence of the Mo substrate and should not be viewed as a simple $\mathrm{SrO}_{x}$ species.

The overall behavior just described, in particular the increase of lattice constant by doping a Sr layer with electronegative oxygen atoms, at first sight seems to be fully compatible with the model of one-dimensional coupling by Friedel oscillations. Oxygen is known to form a fairly strong bond both with metals with the bonding orbital typically several eV below the Fermi level (see, e.g., Ref. 23). This bond formation is generally found to reduce the electron density at the Fermi level, ${ }^{23}$ and to lead finally to the formation of oxides, where this density at $E_{F}$ is zero. A reduction of the electron density at $E_{F}$, on the other hand, leads to an decrease of $k_{F}$ and thus to an increase of the wavelength of the oscillatory part of the potential. Therefore a continuous increase of the lattice constant as a function of oxygen concentration is predicted by this model that may be discretized by the superposition with the strongly site specific local potential of the chemical bond between Sr and Mo that would lead to a sequence of commensurate structures. The absence of any other ordered structure besides $p(8 \times 1)$ and $p(9 \times 1)$ limits the quantitative applicability of this model to only very small concentrations of oxygen. That this model is an over-
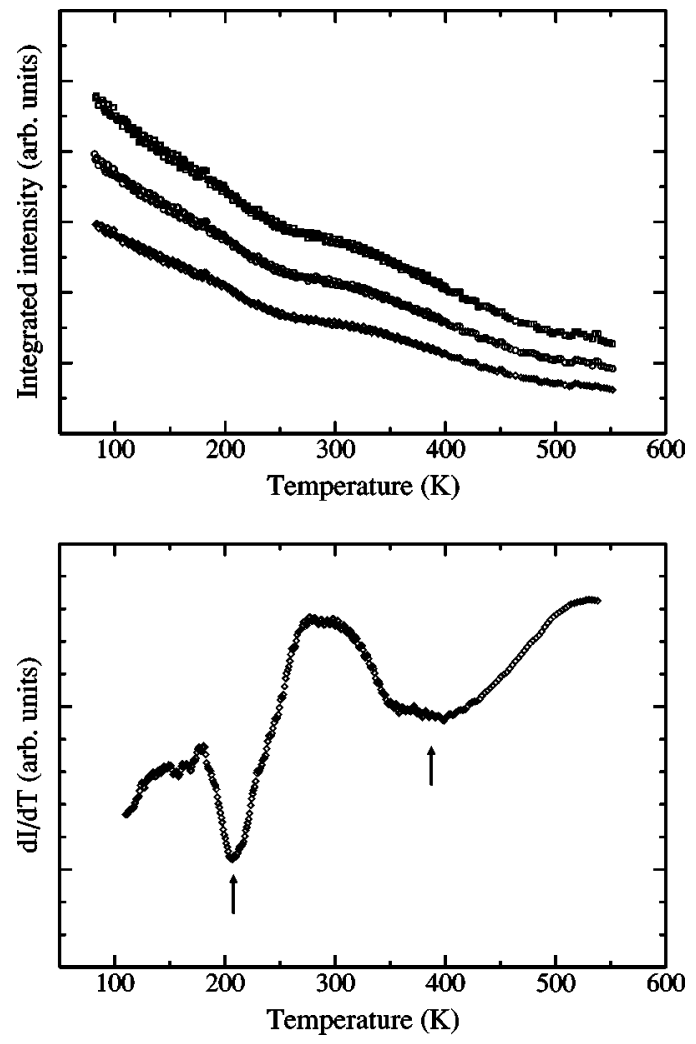

FIG. 6. Top: Example of integrated intensities of $\mathrm{Sr}$ induced superstructure spots with added oxygen as impurity (integration over rectangles with a side ratio of 1:2). The various curves correspond to long sides between 4.3\% BZ (lowest curve) and 6.5\%. The example is taken for the $\left(\frac{\overline{1}}{5} 1\right)$ spot of the $p(5 \times 1)$ structure at a $\mathrm{Sr}$ coverage of $0.2 \mathrm{ML}$ with $0.02 \mathrm{ML}$ of oxygen added. Bottom: Differentiated $4.3 \%$ curve from above after Gaussian smooth. Arrows mark phase transitions.

simplification is also illustrated by our investigations of the Sr- $p(5 \times 1)$ structure desribed in the next section.

\section{B. The effect of oxygen atoms on the $p(5 \times 1)$ structure}

Similar experiments as just described for the $\mathrm{Sr}-p(8 \times 1)$ structure have also been carried out for the $\mathrm{Sr}$ induced $p(5$ $\times 1)$ structure, which is completed at a $\mathrm{Sr}$ coverage of 0.20 . Here we find that up to an additional oxygen concentration of $0.08 \mathrm{ML}$ the average lattice constant of the Sr chain structure is virtually unchanged in both directions at a substrate temperature of $80 \mathrm{~K}$.

First we show in Fig. 6 a representative example of the determination of phase transitions from integrated intensities for the $p(5 \times 1)$ structure at an $\mathrm{Sr}$ coverage of $0.2 \mathrm{ML}$ with the addition of $0.02 \mathrm{ML}$ of oxygen atoms. The two small anomalies both in the integrated intensities and, more pronounced, in the derivative with respect to temperature, which are attributed, as already mentioned, to the depinning transition of the Sr chain lattice and to melting of the chain lattice, are clearly seen.

Contrary to the $p(8 \times 1)$ layer, however, we can still observe a depinning transition in the $\operatorname{Sr}-p(5 \times 1)$ layer for very 


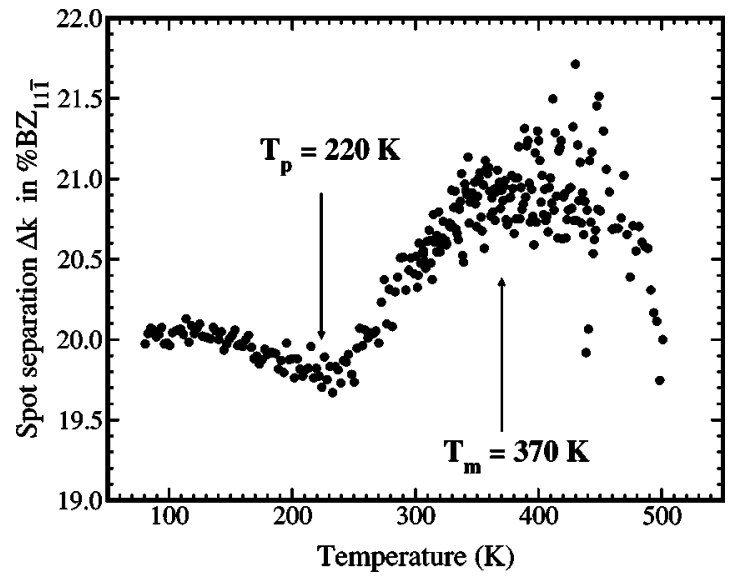

FIG. 7. Distance between $\left(\frac{4}{5} 1\right)$ and ( $\left.\overline{1} 1\right)$ spots as a function of temperature for the $p(5 \times 1)$ structure doped with $0.01 \mathrm{ML}$ of oxygen.

small concentrations of added oxygen ( 0.01 to $0.02 \mathrm{ML}$ ), in contrast to the $p(8 \times 1)$ layer. This difference may be caused by the smaller relative concentration between $\mathrm{Sr}$ and oxygen atoms in the $p(5 \times 1)$ structure at the given concentrations. It emphasizes the extremely strong global effect of oxygen doping in these anisotropic layers.

The change of the average lattice constant in the [1 $\overline{1} 0]$ direction as a function of temperature for a constant $\mathrm{Sr}$ coverage of 0.20 , with $0.01 \mathrm{ML}$ of oxygen added, is shown in Fig. 7. Depinning, found at $220 \mathrm{~K}$ for this concentration of oxygen from the integrated intensities and marked by an arrow, conincides within error bars $( \pm 10 \mathrm{~K})$, as expected, with the onset of a change of the average lattice constant at this concentration of oxygen atoms. The apparent slight shift of the lattice constant in opposite direction at lower temperature is an artifact of our profile fitting routine and not real. Under the influence of the traces of adsorbed oxygen the depinning temperature has more than doubled [ $96 \mathrm{~K}$ for the pure $\mathrm{Sr}$ $-p(5 \times 1)$ layer]. Even more remarkable is the fact that the average lattice constant now shrinks above the depinning temperature, whereas it expanded for the pure $\mathrm{Sr} p(5 \times 1)$ layer. ${ }^{16}$ The melting transition at $380 \mathrm{~K}$ can only be seen approximately in Fig. 7 as the end of the lattice expansion. The precise position was determined from the weak anomaly of the integrated intensity, similar to the pure Sr system. This transition also is shifted upwards in temperature under the influence of oxygen by roughly $150 \mathrm{~K}$.

Depinning and melting transitions are again coupled with characteristic changes of slope in the temperature dependence of the half-widths of the spot profiles (see Fig. 8). At variance with an ideal system, we see a loss of correlation in both measured directions already at the onset of depinning, as indicated by an increase of FWHM with increasing temperature close to $220 \mathrm{~K}$. The melting transition is correlated with an additional increase of slope, more clearly seen in the $(11 \overline{1})$ direction.

The strong stabilization of the $p(5 \times 1)$ structure by doping it with oxygen is demonstrated at various oxygen concentrations, but fixed Sr coverage of 0.20 in the phase dia-

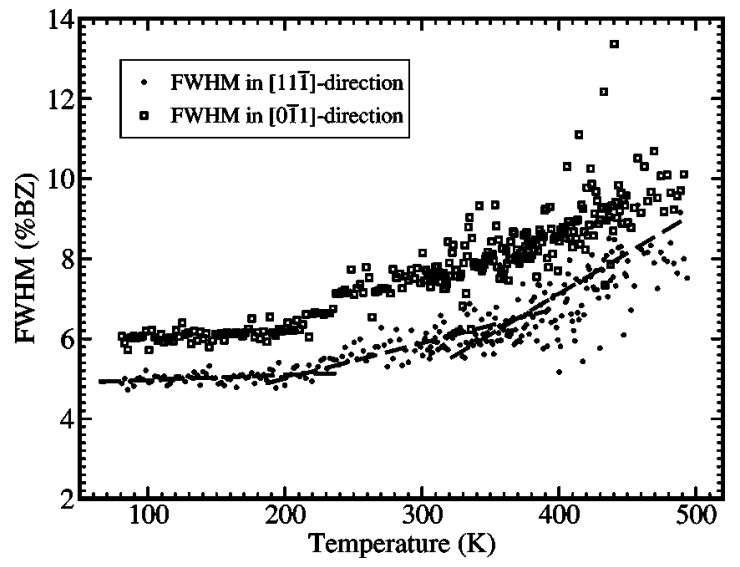

FIG. 8. Temperature dependence of the full widths at half maximum (FWHM), determined for the $\left(\frac{\overline{4}}{5} 1\right)$ superstructure spot of the $\mathrm{Sr}-p(5 \times 1)$ structure at a $\mathrm{Sr}$ coverage of $0.2 \mathrm{ML}$ with $0.01 \mathrm{ML}$ of oxygen added. Data points are for the $(11 \overline{1})$ (circles) and the $(1 \overline{1} 0)$ directions (squares). Dashed lines are only guides to the eye to underline the coupling of the phase transitions of depinning and melting.

gram of Fig. 9. It can be divided into several sections. The first is characterized by simple stabilization of $p(8 \times 1)$ order at low oxygen concentration (up to $\Theta_{o x}=0.04 \mathrm{ML}$ ), with both depinning and melting transitions still observable. Already the smallest oxygen concentration added $(0.01 \mathrm{ML})$ is sufficient, as already described above, to increase both the transitions for depinning and melting by roughly $100 \mathrm{~K}$. A small plateau follows up to an oxygen concentration of about $0.03 \mathrm{ML}$, where the transition temperatures change little as a function of oxygen concentration. Close to $\Theta_{o x}=0.04 \mathrm{ML}$ depinning and melting transitions can no longer be discriminated, and only a melting transition is observed. The behavior of the transitions, in particular the expansion of the lattice upon depinning, remains similiar to the results just described in detail for the oxygen concentration of 0.01 ML.

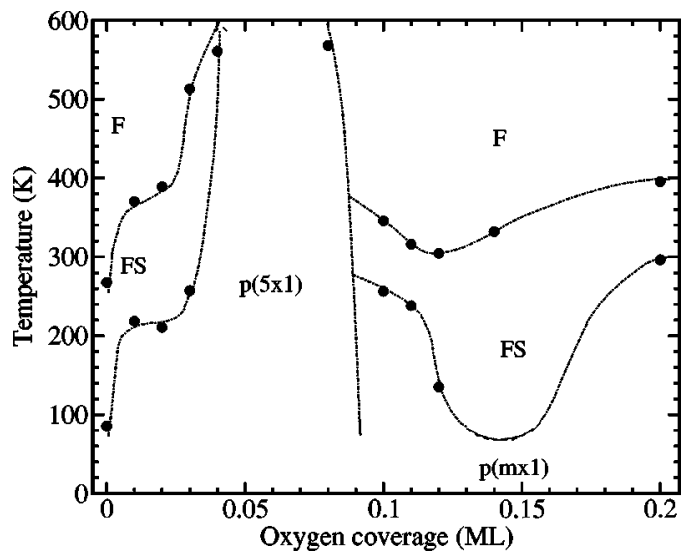

FIG. 9. Phase diagram for $0.2 \mathrm{ML} \mathrm{Sr}$ as a function of added oxygen concentration up to $0.20 \mathrm{ML}$ of oxygen. Black dots represent experimentally measured transition temperatures. Lines mean qualitative observations but are drawn to complement the phase diagram. F means fluid phase, FS floating solid. 


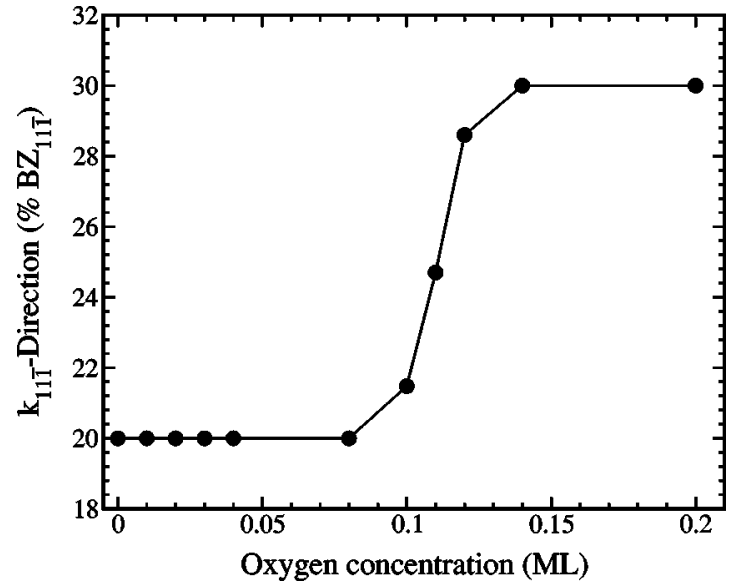

FIG. 10. Change of lattice constant of the $p(5 \times 1)$ structure in the $[11 \overline{1}]$ direction as a function of oxygen concentration. The chains in the perpendicular direction remain close-packed.

In the second section $\left(\Theta_{o x} \leqslant 0.08 \mathrm{ML}\right)$, the stabilization of the $p(5 \times 1)$ structure has reached transition temperatures above $600 \mathrm{~K}$, i.e., they exceed the temperature limit of our equipment for quantitative phase transition measurements. From the steep slope of the phase boundaries in Fig. 9 it may be possible that the melting temperature exceeds even typical temperatures of desorption which set in above $1000 \mathrm{~K}$. Please note that this extreme stabilization of the $p(5 \times 1)$ structure is induced by oxygen concentrations that correspond still to only one oxygen atom per three unit cells of Sr. This means that the global action of the admixture of oxygen is maintained also in this structure. In contrast to the effect of oxygen on the $p(8 \times 1)$ structure, the $p(5 \times 1)$ structure is unchanged by the addition of oxygen atoms, which was unexpected in view of the results at lower $\mathrm{Sr}$ coverage. On the other hand, the oxygen induced stabilization of long range order is observed in both the $p(5 \times 1)$ and the $p(9 \times 1)$ structures. Similar to the observation of the oxygen induced new long range ordered $p(9 \times 1)$ structure, the observation of impurity induced stabilization against thermal disorder is also at variance with the standard models of impurity induced disorder and destruction of phase transitions. ${ }^{24,25} \mathrm{~A}$ second similarity between the results of addition of oxygen to the $p(8 \times 1)$ and $p(5 \times 1)$ structures is that the oxygen atoms themselves are not correlated, since at concentrations below $0.09 \mathrm{ML}$ they do not induce any order that is different from the clean $\operatorname{Sr}-p(5 \times 1)$ layer. The onset of a continuous change of lattice constants above a critical oxygen concentration of $\Theta_{o x} \geqslant 0.09 \mathrm{ML}$ marks the beginning of the third section.

\section{Steps towards oxidation}

As seen from Fig. 10, the $p(5 \times 1)$ structure can indeed be destabilized once a ratio between oxygen and $\mathrm{Sr}$ of roughly 1:2 is reached. At these relatively high concentrations of oxygen it seems that the local bond formation between oxygen and the nearest neighbor $\mathrm{Sr}$ and Mo atoms of the surface dominates. This resembles more an oxidation process than

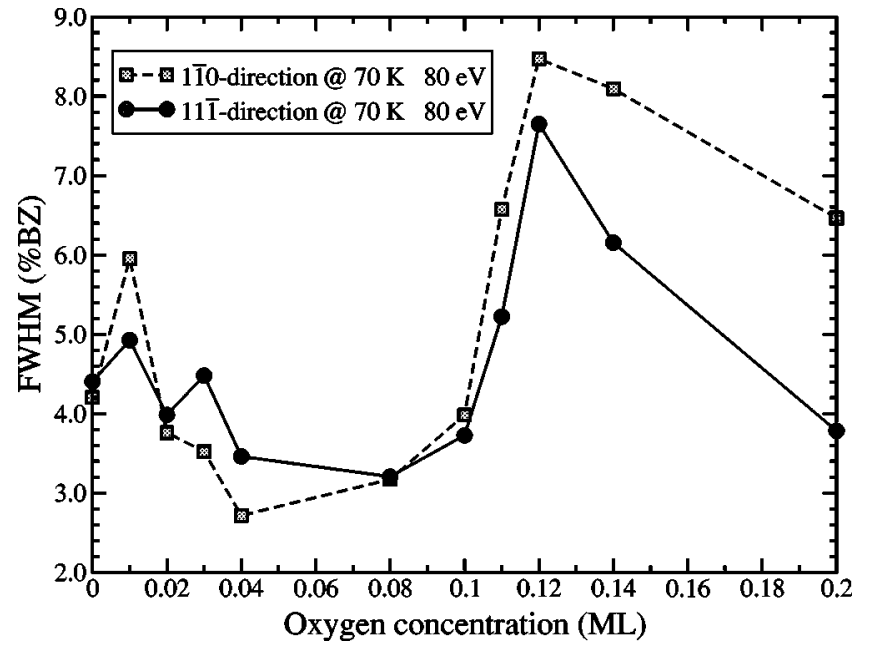

FIG. 11. Half-widths of the $\left(\frac{\overline{4}}{5} 1\right)$ spot, which shifts as a function of oxygen concentration in the range between $0.08<\Theta_{o x}<0.14$ to the spot at the 0.7 position.

what happens with the smaller oxygen concentrations. Therefore we call this part steps towards oxidation.

The average lattice constant starts to be changed by the addition of atomic oxygen above a critical concentration of $\Theta_{o x}=0.08 \mathrm{ML}$. Whereas the close-packed chains remain intact, all superstructure spots are unilaterally and continuously shifted by the additional oxygen adsorption in the $11 \overline{1}$ direction, resulting in incommensurate $(m \times 1)$ structures with incommensurate $m$. This transitional range is rather narrow and ends close to a concentration of $\Theta_{o x}=0.14 \mathrm{ML}$. The structure formed at this and higher oxygen concentrations, still at a constant coverage of $\mathrm{Sr}$ of 0.20 , is still incommensurate with $m=3.3$. This means that a new stable structure is formed. Since the half-widths of the LEED spots as a function of oxygen concentration shown in Fig. 11 get still narrower when further oxygen is added exceeding the concentration of 0.14 , an optimal ratio $\mathrm{O} / \mathrm{Sr}$ for this structure seems to be close to 1:1. At $\Theta_{o x}=0.20 \mathrm{ML}$ the FWHMs are close to those also seen for the pure $\mathrm{Sr}$ layer that seem to be characteristic for the perfectness of the Mo surface.

The $(3.3 \times 1)$ unit cell is most likely a primitive unit cell, containing only one $\mathrm{Sr}$ and one oxygen atom. This follows from the concentration of $\mathrm{Sr}$ available on the surface $(0.2 \mathrm{ML})$ and from the ratio of 1:1. Even with a primitive unit cell, this structure cannot fill the whole surface, i.e., it must form islands. This explains the relatively short correlation lengths observed in this structure. However, this structure, although incommensurate, seems to be the most stable for a $\mathrm{Sr} / \mathrm{O}$ mixture close to a ratio of 1:1 in the whole low coverage regime. Very small islands, giving a very faint LEED pattern, but with the same lattice constant, have also been found for a $\mathrm{Sr}$ coverage of $0.11 \mathrm{ML}$ and similar oxygen concentrations (see the rightmost section of Fig. 2).

Although the exact locations of both $\mathrm{Sr}$ and oxygen atoms can only be determined by, e.g., quantitative LEED, which is under way, some qualitative considerations already reveal some interesting properties. First, the effective nearest neighbor distances between equivalent $\mathrm{SrO}$ units $(9.00 \AA$ in the 
[111] direction, $4.46 \AA$ in the $[1 \overline{1} 0]$ direction) are far off the lattice constant of fcc $\mathrm{SrO}$ (cubic lattice constant $5.139 \AA$ ), underlining the fact that oxygen forms a bond both with $\mathrm{Sr}$ and with the Mo substrate. Second, the transition region between the $p(5 \times 1)$ and the $(3.3 \times 1)$ structure in the oxygen concentration range between $0.08<\Theta<0.14$ exhibits a continuous change of the average lattice constant (see Fig. 10) that is most easily rationalized by the formation of a domain wall network. The local structure in the domain walls corresponds most likely to that of the $(3.3 \times 1)$ structure, i.e., heavy domain walls are formed. This means that $p(5 \times 1)$ and $(3.3 \times 1)$ structures do not demix. Demixing only occurs between $(3.3 \times 1)$ islands and parts of the Mo(112) surface that are essentially empty or have a much lower concentration of $\mathrm{Sr}$ and $\mathrm{O}$.

\section{CONCLUSIONS}

The influence of very small concentrations of oxygen on the long range order in the chain structures of $\mathrm{Sr}$ on $\mathrm{Mo}(112)$ — and presumably also in other systems with similar types of order-is shown in our study not to destroy long range order, as expected for impurities causing a random field-like distortion of the interaction potential. Although the oxygen impurities themselves cannot not be ordered according to our results, they are able to stabilize and even change lateral correlations between the Sr chains. Since the concentrations of oxygen atoms are far below one atom per adsorbate unit cell, the oxygen atoms can only interact globally with the electronic system at the surface. The simplest model how this interaction takes place is by modifications of the adsorbate induced Friedel oscillations screening the adsorbate induced distortion of the electronic system at the surface. ${ }^{26}$ Since the electronic density of states on the $\operatorname{Mo}(112)$ surface is mostly governed by surface states ${ }^{11}$ it can be easily modified by adsorbates, thereby also changing the effective Fermi wavelength at the surface.

Whereas this model can qualitatively describe the behavior observed for the transition between the $p(8 \times 1)$ and $p(9 \times 1)$ structures of $\mathrm{Sr}$, and seems to correlate with the adsorbate induced relaxations in the Mo substrate, ${ }^{9,10}$ a simi- lar transition would be expected for the $p(5 \times 1)$ structure, which is not observed. While this shows the limitations of this simple one-dimensional model applied to an in fact twodimensional system, the global nature of the interaction of oxygen atoms with whole $\mathrm{Sr}$ structure remains valid. The disappearance of the depinning transitions under the influence of oxygen is directly coupled with the observed stabilization effect of the $p(5 \times 1)$ structure, which indicates that also the energies of activation for depinning increase.

It is worth mentioning that the delocalized interaction of oxygen with the $\mathrm{Sr}$ is the still dominating mechanism also at half monolayer coverage of $\mathrm{Sr}^{27}$ where for the pure system a $c(2 \times 2)$ structure is formed, which cannot be described as a chain structure. Depending on concentration, incommensurate $c(2 \times q)$ domain wall structures are formed under the influence of oxygen, finally yielding a $(2 \times 1)$ structure with higher symmetry at a concentration of one oxygen atom per four $\mathrm{Sr}$ unit cells. All these structures can only be explained by explicitly taking into account the full two-dimensional properties of the electronic system.

More local interactions are necessary to explain the structures found at high oxygen concentrations for oxygen to strontium atomic ratios between $1: 2$ and 1:1. While these structures and their properties need further investigations, in particular of their local geometries, it is important to note that these adsorbate systems of electropositive elements on a strongly anisotropic surface like the Mo(112) surface exhibit a surprisingly rich variety and tunability of periodic order. This tunability is even enhanced by doping such layers with electronegative elements like oxygen. From these findings it seems to be attractive to explore the possibilities of using such surfaces as templates for stacks of modulated layers and films. This will be an intriguing task, since also the adsorption of addional layers will modify the electronic system of the original surface thus varying again effective interaction potentials.

\section{ACKNOWLEDGMENTS}

This study was supported by the Deutsche Forschungsgemeinschaft. We thank Dr. I Lyuksyutov and Professor A. Kiejna for helpful discussions.
*Electronic address: pfnuer@fkp.uni-hannover.de

${ }^{1}$ P. Gambardella, M. Blanc, H. Brune, K. Kuhnke, and K. Kern, Phys. Rev. B 61, 2254 (2000).

${ }^{2}$ D. Kolthoff, T. Dullweber, and H. Pfnür, Surf. Sci. 447, 259 (2000).

${ }^{3}$ G. Godzik and H. Pfnür, Surf. Sci. 489, 126 (2001).

${ }^{4}$ H. Yeom, S. Takeda, E. Rotenberg, I. Matsuda, K. Horikoshi, J. Schaefer, C. Lee, S. Kevan, T. Ohta, T. Nagao, and S. Hasegawa, Phys. Rev. Lett. 82, 4898 (1999).

${ }^{5}$ F. Himpsel, K. Altmann, R. Bennewitz, J. Crain, A. Kirakosian, J.-L. Lin, and J. McChesney, J. Phys.: Condens. Matter 13, 11097 (2001)

${ }^{6}$ O. Braun and V. Medvedev, Sov. Phys. Usp. 32, 328 (1989).
${ }^{7}$ D. Kolthoff, H. Pfnür, A. G. Fedorus, and V. Koval, Surf. Sci. 439, 234 (1999).

${ }^{8}$ A. Fedorus, G. Godzik, V. Koval, A. Naumovets, and H. Pfnür, Surf. Sci. 460, 229 (2000).

${ }^{9}$ D. Kolthoff and H. Pfnür, Surf. Sci. 457, 134 (2000).

${ }^{10}$ D. Kolthoff and H. Pfnür, Surf. Sci. 459, 265 (2000).

${ }^{11}$ I. Yakovkin, Surf. Sci. 389, 48 (1997).

${ }^{12}$ G. Grüner, Density Waves in Solids (Addison-Wesley, Redwood City, 1994).

${ }^{13}$ T. Kidd, M. Chou, and T. Chiang, Phys. Rev. Lett. 85, 3684 (2000).

${ }^{14}$ K. Swamy, C. Deisl, A. Menzel, R. Beer, S. Penner, and E. Bertel, Phys. Rev. B 65, 121404 (2002). 
${ }^{15}$ S. Lee, J. Ahn, N. Kim, C. Hwang, J. Chung, H. Yeom, S. Ryikov, and S. Hasegawa, Phys. Rev. Lett. 88, 196401 (2002).

${ }^{16}$ G. Godzik, T. Block, and H. Pfnür, Phys. Rev. B 67, 125424 (2003).

${ }^{17}$ I. Lyuksyutov, A. Naumovets, and V. Pokrovsky, TwoDimensional Crystals (Academic, Boston, 1992).

${ }^{18}$ D. Jürgens, G. Held, and H. Pfnür, Surf. Sci. 303, 77 (1994).

${ }^{19}$ A. Fedorus, D. Kolthoff, V. Koval, I. Lyuksyuotov, A. Naumovets, and H. Pfnür, Phys. Rev. B 62, 2852 (2000).

${ }^{20}$ K. Fukui, T. Aruga, and Y. Iwasawa, Surf. Sci. 281, 241 (1993).

${ }^{21}$ A. Santra, B. Min, and D. Goodman, Surf. Sci. 513, L441 (2002).
${ }^{22}$ V. Gavriluk and V. Medvedev, Sov. Phys. Solid State 8, 1439 (1966).

${ }^{23}$ L. Savio, L. Vatttone, M. Rocca, V. D. Renzi, S. Gardonio, C. Mariani, U. del Pennino, G. Cipriani, A. D. Corso, and S. Baroni, Surf. Sci. 486, 65 (2001).

${ }^{24}$ A. Harris, J. Phys. C 7, 1671 (1974).

${ }^{25}$ L. Schwenger, K. Budde, C. Voges, and H. Pfnür, Phys. Rev. Lett. 73, 296 (1994).

${ }^{26}$ B. Gumhalter and W. Brenig, Surf. Sci. 336, 326 (1995).

${ }^{27}$ G. Godzik, H. Pfnür, and I. Lyuksyutov, Europhys. Lett. 56, 67 (2001). 